\title{
Hazardous chemicals: psychological dimensions of the health sequelae of a community exposure in Texas
}

\author{
Hari H Dayal, Tom Baranowski, Yi-hwei Li, Ralph Morris
}

\begin{abstract}
Study objective - A chemical spill from an oil refinery exposed the local community to more than $40000 \mathrm{lb}$ of highly toxic and corrosive hydrofluoric acid. A community based symptom prevalence study found an association between exposure and physical symptoms: the psychological impact of the disaster and its potential effect on the reporting of physical symptoms is examined here.
\end{abstract}

Design - The study used a population based survey design consisting of two phases: phase I, the exposure phase, and, phase II, the symptom prevalence phase. Subjects - In phase I, information indicative of exposure was collected on 10811 individuals in a door to door survey of a geographically defined area. In phase II, symptom prevalence information was gathered through in-person interviews with 2509 subjects selected from the phase I census in a sampling scheme that balanced across the exposure categories with regard to age, gender, and predisposition. The refusal rate in both phases was $<5 \%$.

Measurement and main results - Stepwise logistic regression analyses were used to assess the relative predictive importance of psychological variables and hydrofluoric acid exposure in explaining the reported physical symptoms two years after the disaster. The findings show a linear relationship between the level of hydrofluoric acid exposure and the degree of psychological stress two years after the accident. High exposure had a long term (more than two years) impact on physical health for some self reported symptoms, even after controlling for the psychological impact. Some physical symptom reports, however, were better explained by psychological status than by exposure to hydrofluoric acid. The physical symptoms for which exposure was the major predictor were those for which the biological plausibility of a relationship with hydrofluoric acid exposure was direct.

University of Texas, Medical Branch of Galveston, Route J-09,
Galveston, Texas 77555 1009, USA

H H Dayal

T Baranowski

$\mathrm{T} \mathrm{Li}$

Galveston County Health District, Galveston, Texas, USA R Morris

Correspondence to: Dr H H Dayal

Accepted for publication March 1994
Anhydrous hydrogen fluoride (HF) is a highly irritating and corrosive substance because of its high solubility in water and the capability of concentrated HF solutions to absorb water with a release of heat. These properties mean that it can cause severe burns to the skin and eyes as well as pulmonary damage, which may not be painful or visible for several hours after the initial exposure. ${ }^{1}$ Once absorbed, HF has some direct and indirect systemic toxic effects which occur as a result of the rise of the fluoride concentration in the blood (direct), and the concomitant lowering of calcium and magnesium in blood, skin, and other organs (indirect). Considerable local and systemic toxicity may occur from hydrofluoric acid by all routes of exposure. ${ }^{2}$ Published literature on toxicity of fluoride has mainly dealt with chronic (low dose, long term) exposure..$^{3-5}$

Acute exposures to HF have been primarily investigated in occupational settings. Pulmonary oedema and death within a couple of hours of inhaling HF have been reported. ${ }^{6-8} \mathrm{~A}$ case of pulmonary injury manifesting several days after exposure and death in four weeks has also been reported. ${ }^{9}$ Respiratory symptoms may persist for months to years after inhalation injury. ${ }^{9}$ Exposure of the eye to HF produces more extensive damage than that of other acids in similar concentrations. ${ }^{2}$ Dermal exposure causes severe skin burns and intense pain, the onset of which may be delayed for hours. Severe burns can progress to ulceration and tissue loss, which, in extreme cases, may result in scarring and permanent disability. ${ }^{1011}$ There are also reports of systemic fluoride toxicity from acute HF exposure: Tepperman, ${ }^{12}$ for example, reports a fatality resulting from a severe facial burn, which produced acute systemic fluoride poisoning. Other reports of systemic toxicity from acute exposures include systemic acidosis, hypocalcaemia and hypomagnesia, ${ }^{13}$ and histological myocardial damage. ${ }^{14}$

On October 30, 1987, the convection section of the HF alkylation heater was dropped during an attempted crane lift at the Marathon Oil refineries in Texas City, Texas. Two relief valves were sheared off a storage tank and over $40000 \mathrm{lb}$ of anhydrous HF and 200-300 barrels of isobutane were released into the atmosphere, forming a huge cloud that drifted over residential areas of the community. Residents (approximately 200 blocks) were evacuated for $\mathbf{4 8}$ hours. After the spill, about 1000 people were seen at the emergency department of the two area hospitals; 95 were considered sick enough to be admitted to hospital. The Texas City episode was the first substantial acute HF exposure involving an entire community.

A population based symptom survey was 
conducted to evaluate the accident's impact on the health of the community. Details of the methods and findings with regard to short term and long term physical symptoms have been published. ${ }^{15}$ The principle findings with regard to physical symptoms can be summarised as follows: (i) the prevalence of severe individual physical symptoms (and the prevalence of severe symptoms in an organ/function system) in the month after the spill increased in relation to exposure in a dose-response manner; (ii) the continuing prevalence of severe individual symptoms (and prevalence of severe symptoms in an organ/function system) two years after the spill was also related to the degree of exposure; (iii) the reported prevalence of severe symptoms two years after the spill was considerably lower than the prevalence in the month after the spill in each exposure category, and the improvement was most substantial for the highly exposed group; and (iv) symptoms like skin burns and blistering were mostly short term, even in the highly exposed individuals, whereas problems related to breathing were reported to be still severe two years after the spill by 20 to $30 \%$ of the individuals in the highly exposed group. Thus, Dayal et $a l^{15}$ showed that exposure produced short and long term adverse physical symptoms in the community.

Disasters have also been shown to have long term psychological effects, ${ }^{16-19}$ and people with psychological problems or stress tend to report more symptoms of any kind. ${ }^{20}$ Thus, the long term psychological impact of the accident could potentially account for the higher frequency of physical symptoms in the exposed groups. We examine here the interrelationships of the physical and psychological dimensions of the health sequelae of the community exposure to $\mathrm{HF}$ in Texas City. The relative predictive value of the psychological variables and $\mathrm{HF}$ exposure in explaining physical symptoms is ascertained through multivariate analyses.

\footnotetext{
Methods

The population based epidemiological study comprised two phases. Phase I, the exposure study, was a door to door survey of 10811 individuals in 339 city blocks conducted almost a year after the spill to obtain a census of individuals who had come into contact with the chemical and to assign a measure of exposure to each person in the list. We adopted a multipronged approach as we recognised that there would be uncertainty associated with any single measure. Data were gathered on the whereabouts of each person at 15 minute intervals for the two hours after the release, a respondent reported level of exposure, and, for the 48 hour period afterwards, an assessment was made of the severity of the three acute symptoms considered pathogonomonic - burning or irritation of the throat, burning or irritation of eyes, and coughing or difficulty breathing. In addition, predisposition to lung problems was designated for individuals with a pre-existing pulmonary condition or those who smoked two
}

or more packets of cigarettes per day at the time of the spill.

A mathematical model was used to predict isodensity curves of $\mathrm{HF}$ concentrations at various locations at specific times. The model predicted concentrations on a 400 point grid, and this grid was used to draw concentration isopleths for each of the first two hours and for the two hour average. A cumulative exposure index was produced by relating the whereabouts data to projected concentration levels. The model predicted exposure index and the other two indicators exhibited very poor correlation, even when ranks were used. To gain further insight, the location of isodensity curves was compared to the residence of the patients admitted to hospital, and the residence location of those considered to have been heavily exposed by the two highly correlated indicators. It was significant that the cluster of patients admitted to hospital (objectively known to be highly exposed) conformed to the cluster of individuals designated as heavily exposed by the self reports, and neither the hospitalised cluster nor the cluster of designated heavily exposed individuals was consistent with the areas identified by the models as areas of high exposure. These observations suggested the severe limitations of the mathematical modelling approach in defining individual doses from a brief exposure. In fact, the lack of detailed meteorological data and the acute nature of the exposure made the mathematical modelling approach inadmissible.

The selected method of exposure assessment in this study was the combination of the overall reported level and the data on severity of the three pathogonomonic acute symptoms associated with the exposure. The questions on acute symptoms were omitted for individuals for whom exposure was reported as 'none' or as 'don't know'. For others, the information on acute symptoms was summarised into none reported, none severe, one severe, two severe, and all three severe. The data on the reported level of exposure and severe acute symptoms were combined according to the schema presented in the figure, to yield the following exposure categories: high, moderate, mild, none, not well defined, and unknown. Later, the 'mild' and 'moderate' categories were merged to create an 'intermediate' exposure category. It should be noted that pathogonomonic symptoms reported at mild or moderate levels did not enter the final designation of an individual's exposure category. Overall, $6 \cdot 7 \%$ were determined to have been heavily exposed, and $51.3 \%$ reported no exposure.

Phase II, the symptom prevalence survey, was conducted approximately two years after the accident and consisted of face to face interviews with 2509 people. This included all highly exposed individuals from the exposure study and a sample of controls from the census. The simple random sampling of controls was restricted in a way to produce balance across the exposure categories with regard to age, gender, and predisposition. Complete data were collected from 1994 of the 2509 in- 

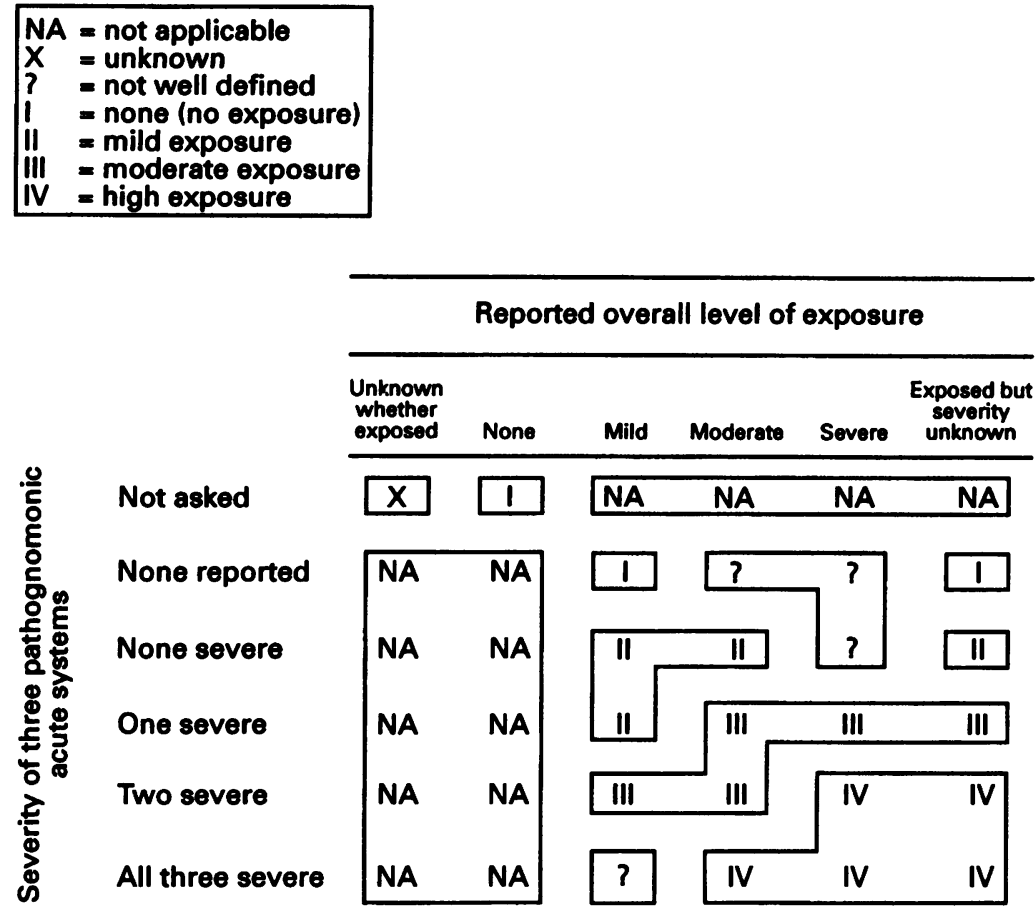

Designation of exposure categories based on combinations of reported overall level of exposure versus the severity of three characteristic acute symptoms.

dividuals sampled, the completion rates being $78 \%, 80 \%$, and $80 \%$ for the high, intermediate, and no exposure groups respectively.

The symptom prevalence questionnaire included items on the presence and severity of 40 physical symptoms in the month after the spill, and, for the initially reported symptoms, their presence and severity in the past month. The 40 physical symptoms were grouped into nine organ/function systems - throat, stomach, chest, skin, nose, breathing, eye, problems waking up at night, and miscellaneous (dizziness, trouble concentrating, tingling or numbness). Based on the known chemical properties of $\mathrm{HF}$, some of these symptom groups were more likely to be causally related to a physical exposure than others.

A list of 33 psychological symptoms (see Appendix) was used to assess the psychological effect in the week preceding the interview in subjects 17 years of age or older. The list was derived from the Brief Symptom Inventory (BSI) of 53 symptoms, which is a version of SCL-90, ${ }^{21}$ shortened by deleting four constructs that were unlikely to result from an acute event, that is obsessive-compulsive, interpersonal sensitivity, phobic anxiety, and psychoticism. Thus, the selected constructs represented by the 33 symptoms include: somatisation, depression, anxiety, hostility, and paranoia. The correlations between the SCL90 and BSI for the nine constructs range from 0.92 to $0.99 .^{22}$ The severity of each symptom was recorded as none, a little bit, quite a bit, a whole lot, and don't know, and responses from 'none' to 'a whole lot' were coded 0 to 3 . After the authors' recommendations, ${ }^{22}$ a summary psychological score ranging from 0 to 99 was created for each respondent by adding the intensity values of perceived distress from each symptom. Individuals with more than six 'don't knows' were excluded from analysis; up to six 'don't knows' were normalised into the known responses by weighting the score by a factor reflecting the proportion of symptoms with a known intensity. Summary construct scores were similarly generated for each of the five constructs.

So that time frames for the physical and psychological symptoms were comparable, only long term physical symptoms (that is, symptoms reported to continue in the month preceding the interview) are considered in this paper. Contingency table analyses were conducted to discern associations. Measures of association are presented in terms of the Gamma statistic, which is appropriate for ordinal data with ties. Multivariate analyses used a stepwise logistic regression procedure. The magnitude of the standardised coefficient associated with a factor was taken to be an indicator of its importance in predicting the outcome - that is, a severe individual symptom or a severe symptom in an organ/function system. The goodness of fit of the final model was assessed using a $\chi^{2}$ test that compared the observed and expected decile distributions. ${ }^{23}$ As such, a small $\chi^{2}$ statistic, and, correspondingly a large $p$ value was indicative of a large $p$ value was indicative of a good agreement between the observed outcomes and those predicted by the final model.

Separate logistic regression analyses were run for each of the nine organ/function systems. The dichotomous dependent variable in these analyses was the report of a severe symptom in the system, or not. Explanatory factors offered in the model included: exposure entered as two design (dummy) variables to describe the three levels (none, intermediate, high); age entered as a continuous variable; total psychological score entered as a continuous variable; gender entered as a categorical variable; predisposition entered as a categorical variable; and the two terms describing the interaction between the psychological score and exposure. To enter the model, a variable had to be significant at a level of $p=0.05$. Since the main issue in this article was the relative explanatory importance of psychological status versus the exposure, however, these factors were forced into the model. In subsequent logistic regression analyses, instead of the total psychological score, summary scores for individual constructs were used as continuous variables. As would be expected, somatisation dominated as a predictor. In fact, no other psychological construct was selected in the models. Since we wished to examine other psychological determinants of long term health effects, we then conducted analyses in which somatisation was not offered. This decision was not therefore a statistical decision. Finally, analyses with the total psychological score as well as the four individual construct scores were repeated for selected individual physical symptoms. The individual symptoms chosen for these analyses represented an array of physical effects ranging from those that offered the most direct biological plausibility for a causal association with the exposure (that 
Table 1 Psychological symptom score in relation to exposure category

\begin{tabular}{|c|c|c|c|c|}
\hline \multirow[t]{2}{*}{ Symptom score } & \multicolumn{3}{|c|}{ Exposure category } & \multirow{2}{*}{$\begin{array}{l}\text { Combined sample } \\
(n=1343)(\%)\end{array}$} \\
\hline & $\begin{array}{l}\text { None } \\
(n=472) \\
(\%)\end{array}$ & $\begin{array}{l}\text { Intermediate } \\
(n=465) \\
(\%)\end{array}$ & $\begin{array}{l}\text { High } \\
(n=406) \\
(\%)\end{array}$ & \\
\hline $\begin{array}{l}0 \\
1-5 \\
6-15 \\
16-99\end{array}$ & $\begin{array}{l}29 \cdot 03 \\
34 \cdot 53 \\
21 \cdot 19 \\
15 \cdot 25\end{array}$ & $\begin{array}{l}14 \cdot 62 \\
23.66 \\
29.03 \\
32.69\end{array}$ & $\begin{array}{l}10.59 \\
13.05 \\
24.63 \\
51.72\end{array}$ & $\begin{array}{l}18 \cdot 5 \\
24 \cdot 2 \\
25 \cdot 0 \\
32 \cdot 3\end{array}$ \\
\hline
\end{tabular}

Gamma $=0.428$ sample, ensuring adequate numbers in each category. The proportion of individuals with a higher psychological score increased with exposure. The Gamma measure of association was $0.428(\mathrm{p}=0.02)$.

Table 2 presents the results of stepwise logistic regression analyses for the various organ/ function systems' symptoms. The psychological score $\mathrm{X}$ exposure interaction term did not enter the final model for any of the nine systems. Similarly, gender was not a predictor of a severe symptom in any organ/function system. Age entered the final model for all systems except the constellation of symptoms related to stomach and skin, and a positive estimated coefficient indicated that there was a higher probability of a severe symptom in those systems among older people after adjustment had been made for all the other factors offered in the model. High HF exposure was the most important predictor of a severe symptom in all organ/function systems. Intermediate exposure was at least as important a predictor of a continuing severe physical symptom as the psychological score for those organ/function systems most likely directly related to the exposure, that is, throat, skin, nose, breathing, and eyes. The total psychological score was a relatively important predictor of a severe symptom for organ/function systems less likely to have a direct relationship to the exposure, that is, stomach, chest, problems that wake one up at night, and the miscellaneous problems.

Table 3 presents the predictors of selected individual symptoms. The psychological score

Table 2 Predictors of severe physical symptoms in organ/function systems with total psychological score*

\begin{tabular}{|c|c|c|c|c|c|c|}
\hline $\begin{array}{l}\text { Organ/function } \\
\text { system } \dagger\end{array}$ & $\begin{array}{l}p \text { value for } \\
\text { goodness of fit }\end{array}$ & $\begin{array}{l}\text { Predictors entering } \\
\text { the model } \neq\end{array}$ & $\hat{\beta}$ & $S E(\hat{\beta})$ & $p$ & $\begin{array}{l}\text { Standardised } \\
\text { estimate }\end{array}$ \\
\hline Throat & 0.1246 & $\begin{array}{l}\text { Exposure (inter) } \\
\text { Exposure (high) } \\
\text { Psych score } \\
\text { Age }\end{array}$ & $\begin{array}{l}0.91 \\
1.84 \\
0 \cdot 02 \\
0 \cdot 01\end{array}$ & $\begin{array}{l}0 \cdot 249 \\
0 \cdot 241 \\
0 \cdot 004 \\
0 \cdot 004\end{array}$ & $\begin{array}{l}0.0003 \\
0.0001 \\
0.0001 \\
0.0233\end{array}$ & $\begin{array}{l}0 \cdot 24 \\
0 \cdot 47 \\
0 \cdot 15 \\
0 \cdot 10\end{array}$ \\
\hline Stomach & $0 \cdot 8047$ & $\begin{array}{l}\text { Exposure (inter) } \\
\text { Exposure (high) } \\
\text { Psych score }\end{array}$ & $\begin{array}{l}0.55 \\
1.55 \\
0.04\end{array}$ & $\begin{array}{l}0.393 \\
0.363 \\
0.005\end{array}$ & $\begin{array}{l}0 \cdot 1596 \\
0 \cdot 0001 \\
0 \cdot 0001\end{array}$ & $\begin{array}{l}0 \cdot 14 \\
0 \cdot 39 \\
0 \cdot 37\end{array}$ \\
\hline Chest & 0.4394 & $\begin{array}{l}\text { Exposure (inter) } \\
\text { Exposure (high) } \\
\text { Psych score } \\
\text { Age }\end{array}$ & $\begin{array}{l}0.75 \\
1 \cdot 84 \\
0 \cdot 04 \\
0 \cdot 02\end{array}$ & $\begin{array}{l}0.339 \\
0.318 \\
0.005 \\
0.006\end{array}$ & $\begin{array}{l}0.0279 \\
0.0001 \\
0.0001 \\
0.0001\end{array}$ & $\begin{array}{l}0 \cdot 20 \\
0 \cdot 47 \\
0 \cdot 34 \\
0 \cdot 23\end{array}$ \\
\hline Skin & $0 \cdot 1272$ & $\begin{array}{l}\text { Exposure (inter) } \\
\text { Exposure (high) } \\
\text { Psych score }\end{array}$ & $\begin{array}{l}0.69 \\
1.83 \\
0.02\end{array}$ & $\begin{array}{l}0 \cdot 304 \\
0 \cdot 282 \\
0 \cdot 004\end{array}$ & $\begin{array}{l}0 \cdot 0233 \\
0 \cdot 0001 \\
0 \cdot 0001\end{array}$ & $\begin{array}{l}0 \cdot 18 \\
0 \cdot 46 \\
0 \cdot 19\end{array}$ \\
\hline Nose & $0 \cdot 3750$ & $\begin{array}{l}\text { Exposure (inter) } \\
\text { Exposure (high) } \\
\text { Psych score } \\
\text { Age }\end{array}$ & $\begin{array}{l}0.85 \\
1.56 \\
0.02 \\
0.01\end{array}$ & $\begin{array}{l}0 \cdot 255 \\
0 \cdot 249 \\
0 \cdot 004 \\
0 \cdot 005\end{array}$ & $\begin{array}{l}0.0009 \\
0.0001 \\
0.0001 \\
0.0029\end{array}$ & $\begin{array}{l}0 \cdot 22 \\
0 \cdot 40 \\
0 \cdot 20 \\
0 \cdot 13\end{array}$ \\
\hline Breathing & $0 \cdot 0194$ & $\begin{array}{l}\text { Exposure (inter) } \\
\text { Exposure (high) } \\
\text { Psych score } \\
\text { Age }\end{array}$ & $\begin{array}{l}0.86 \\
1.69 \\
0.03 \\
0.01\end{array}$ & $\begin{array}{l}0 \cdot 198 \\
0 \cdot 196 \\
0 \cdot 004 \\
0 \cdot 004\end{array}$ & $\begin{array}{l}0.0001 \\
0.0001 \\
0.0001 \\
0.0002\end{array}$ & $\begin{array}{l}0 \cdot 23 \\
0 \cdot 43 \\
0 \cdot 25 \\
0 \cdot 14\end{array}$ \\
\hline Eye & 0.6869 & $\begin{array}{l}\text { Exposure (inter) } \\
\text { Exposure (high) } \\
\text { Psych score } \\
\text { Age }\end{array}$ & $\begin{array}{l}0.91 \\
2.06 \\
0.03 \\
0.01\end{array}$ & $\begin{array}{l}0 \cdot 226 \\
0 \cdot 218 \\
0.004 \\
0.004\end{array}$ & $\begin{array}{l}0.0001 \\
0.0001 \\
0.0001 \\
0.0192\end{array}$ & $\begin{array}{l}0 \cdot 24 \\
0 \cdot 52 \\
0 \cdot 25 \\
0 \cdot 09\end{array}$ \\
\hline Wake up & 0.4593 & $\begin{array}{l}\text { Exposure (inter) } \\
\text { Exposure (high) } \\
\text { Psych score } \\
\text { Age } \\
\text { Predisposition }\end{array}$ & $\begin{array}{l}0.56 \\
1.51 \\
0.03 \\
0.01 \\
0.37\end{array}$ & $\begin{array}{l}0 \cdot 215 \\
0 \cdot 207 \\
0 \cdot 004 \\
0 \cdot 004 \\
0 \cdot 167\end{array}$ & $\begin{array}{l}0.0092 \\
0.0001 \\
0.0001 \\
0.0006 \\
0.0257\end{array}$ & $\begin{array}{l}0 \cdot 15 \\
0.38 \\
0 \cdot 32 \\
0 \cdot 14 \\
0.09\end{array}$ \\
\hline Miscellaneous & $0 \cdot 0884$ & $\begin{array}{l}\text { Exposure (inter) } \\
\text { Exposure (high) } \\
\text { Psych score } \\
\text { Age }\end{array}$ & $\begin{array}{l}1 \cdot 49 \\
2 \cdot 12 \\
0.05 \\
0.03\end{array}$ & $\begin{array}{l}0.348 \\
0.341 \\
0.005 \\
0.005\end{array}$ & $\begin{array}{l}0.0001 \\
0.0001 \\
0.0001 \\
0.0001\end{array}$ & $\begin{array}{l}0.39 \\
0.54 \\
0.48 \\
0.29\end{array}$ \\
\hline
\end{tabular}

${ }^{*}$ Results based on stepwise logistic regression analysis.

† The dichotomous outcome is at least one symptom rated as severe in the organ/function system continuing two years past the spill.

$\ddagger$ The factors, Exposure (inter), Exposure (high), and Psych score, were forced into the model. The other independent variables offered in models were Age, Gender, Predisposition, Exposure (inter) $\times$ Psych score, and Exposure (high) $\times$ Psych score and they were selected stepwise at the 0.05 level. 
Table 3 Predictors of severe individual symptoms with total psychological score*

\begin{tabular}{|c|c|c|c|c|c|c|}
\hline Symptom & $\begin{array}{l}\text { p value for } \\
\text { the goodness } \\
\text { of fit }\end{array}$ & $\begin{array}{l}\text { Predictors entering } \\
\text { the model } \neq\end{array}$ & $\hat{\hat{\beta}}$ & $S E(\hat{\beta})$ & $p$ & $\begin{array}{l}\text { Standardised } \\
\text { estimate }\end{array}$ \\
\hline Phlegm & $0 \cdot 1120$ & $\begin{array}{l}\text { Exposure (inter) } \\
\text { Exposure (high) } \\
\text { Psych score } \\
\text { Age }\end{array}$ & $\begin{array}{l}0.52 \\
1.50 \\
0.01 \\
0.01\end{array}$ & $\begin{array}{l}0.279 \\
0.263 \\
0.005 \\
0.005\end{array}$ & $\begin{array}{l}0 \cdot 0642 \\
0 \cdot 0001 \\
0 \cdot 0411 \\
0 \cdot 0138\end{array}$ & $\begin{array}{l}0 \cdot 14 \\
0.38 \\
0 \cdot 09 \\
0 \cdot 12\end{array}$ \\
\hline Diarrhoea & 0.6144 & $\begin{array}{l}\text { Exposure (inter) } \\
\text { Exposure (high) } \\
\text { Psych score } \\
\text { Exposure (high) x } \\
\text { Psych score }\end{array}$ & $\begin{array}{r}-0.43 \\
2.32 \\
0.06 \\
-0.05\end{array}$ & $\begin{array}{l}0.561 \\
0.549 \\
0.011 \\
0.014\end{array}$ & $\begin{array}{l}0 \cdot 4407 \\
0 \cdot 0001 \\
0 \cdot 0001 \\
0 \cdot 0005\end{array}$ & $\begin{array}{r}-0.11 \\
0.59 \\
0.55 \\
-0.40\end{array}$ \\
\hline $\begin{array}{l}\text { Shortness of } \\
\text { breath }\end{array}$ & 0.6806 & $\begin{array}{l}\text { Exposure (inter.) } \\
\text { Exposure (high) } \\
\text { Psych score } \\
\text { Age } \\
\text { Predisposition }\end{array}$ & $\begin{array}{l}0.59 \\
2.07 \\
0.03 \\
0.02 \\
0.75\end{array}$ & $\begin{array}{l}0 \cdot 337 \\
0 \cdot 307 \\
0 \cdot 005 \\
0 \cdot 006 \\
0 \cdot 204\end{array}$ & $\begin{array}{l}0.0806 \\
0.0001 \\
0 \cdot 0001 \\
0 \cdot 0001 \\
0 \cdot 0002\end{array}$ & $\begin{array}{l}0 \cdot 15 \\
0.52 \\
0 \cdot 25 \\
0 \cdot 21 \\
0 \cdot 18\end{array}$ \\
\hline Headaches & 0.3003 & $\begin{array}{l}\text { Exposure (inter) } \\
\text { Exposure (high) } \\
\text { Psych score } \\
\text { Gender }\end{array}$ & $\begin{array}{l}0.43 \\
1.49 \\
0 \cdot 03 \\
0 \cdot 44\end{array}$ & $\begin{array}{l}0 \cdot 252 \\
0 \cdot 236 \\
0 \cdot 004 \\
0 \cdot 178\end{array}$ & $\begin{array}{l}0 \cdot 0895 \\
0 \cdot 0001 \\
0 \cdot 0001 \\
0 \cdot 0136\end{array}$ & $\begin{array}{l}0 \cdot 11 \\
0 \cdot 38 \\
0 \cdot 30 \\
0 \cdot 12\end{array}$ \\
\hline $\begin{array}{l}\text { Trouble } \\
\text { concentrating }\end{array}$ & $0 \cdot 7480$ & $\begin{array}{l}\text { Exposure (inter) } \\
\text { Exposure (high) } \\
\text { Psych score } \\
\text { Age }\end{array}$ & $\begin{array}{l}1.00 \\
1.17 \\
0.06 \\
0.03\end{array}$ & $\begin{array}{l}0.488 \\
0.481 \\
0.006 \\
0.008\end{array}$ & $\begin{array}{l}0 \cdot 0400 \\
0 \cdot 0152 \\
0 \cdot 0001 \\
0 \cdot 0002\end{array}$ & $\begin{array}{l}0 \cdot 26 \\
0 \cdot 30 \\
0 \cdot 61 \\
0 \cdot 29\end{array}$ \\
\hline
\end{tabular}

* Results are based on stepwise logistic regression analysis.

t The dichotomous outcome is severe symptom continuing two years past the spill.

$¥$ The factors, Exposures (inter), Exposure (high), and Psych score, were forced into models. The other independent variables offered in models were age, gender, predisposition, exposure (inter) $\times$ Psych score, and Exposure (high) $\times$ Psych score; and they were selected stepwise at the 0.05 level.

$\mathrm{X}$ exposure interaction term entered the model only for diarrhoea. Gender entered the final prediction model for headache as females have a higher probability of reporting a severe headache than males. High HF exposure was the most important predictor of symptoms of phlegm and shortness of breath. For diarrhoea and headaches, the high $\mathrm{HF}$ exposure and psychological status seemed to be of roughly equal importance. However, the total psychological score was the prominent predictor of the symptom of trouble in concentrating.

Results of logistic regression analyses in which individual psychological constructs were

Table 4 Predictors of severe physical symptoms in organ function systems with individual psychological construct scores*

\begin{tabular}{|c|c|c|c|c|c|c|}
\hline $\begin{array}{l}\text { Organ function } \\
\text { system } \dagger\end{array}$ & $\begin{array}{l}\text { p value for } \\
\text { the goodness } \\
\text { of fit }\end{array}$ & $\begin{array}{l}\text { Predictors entering } \\
\text { the model } \neq\end{array}$ & $\hat{\beta}$ & $S E(\hat{\beta})$ & $p$ & $\begin{array}{l}\text { Standardised } \\
\text { estimate }\end{array}$ \\
\hline Throat & 0.4033 & $\begin{array}{l}\text { Exposure (inter) } \\
\text { Exposure (high) } \\
\text { Anxiety } \\
\text { Hostility } \\
\text { Predisposition }\end{array}$ & $\begin{array}{r}0.94 \\
1.91 \\
0 \cdot 14 \\
-0.13 \\
0.38\end{array}$ & $\begin{array}{l}0 \cdot 249 \\
0 \cdot 239 \\
0 \cdot 028 \\
0 \cdot 040 \\
0 \cdot 179\end{array}$ & $\begin{array}{l}0.0002 \\
0.0001 \\
0.0001 \\
0.0011 \\
0.0332\end{array}$ & $\begin{array}{r}0 \cdot 25 \\
0 \cdot 48 \\
0 \cdot 28 \\
-0 \cdot 20 \\
0 \cdot 09\end{array}$ \\
\hline Stomach & 0.4493 & $\begin{array}{l}\text { Exposure (inter) } \\
\text { Exposure (high) } \\
\text { Anxiety }\end{array}$ & $\begin{array}{l}0.62 \\
1.68 \\
0 \cdot 17\end{array}$ & $\begin{array}{l}0.391 \\
0.359 \\
0.023\end{array}$ & $\begin{array}{l}0 \cdot 1108 \\
0 \cdot 0001 \\
0 \cdot 0001\end{array}$ & $\begin{array}{l}0.16 \\
0.43 \\
0.33\end{array}$ \\
\hline Chest & 0.2526 & $\begin{array}{l}\text { Exposure (inter) } \\
\text { Exposure (high) } \\
\text { Anxiety } \\
\text { Age }\end{array}$ & $\begin{array}{l}0 \cdot 80 \\
1.96 \\
0 \cdot 15 \\
0 \cdot 02\end{array}$ & $\begin{array}{l}0.338 \\
0.314 \\
0.022 \\
0.006\end{array}$ & $\begin{array}{l}0.0174 \\
0.0001 \\
0.0001 \\
0.0001\end{array}$ & $\begin{array}{l}0 \cdot 21 \\
0 \cdot 50 \\
0 \cdot 30 \\
0 \cdot 23\end{array}$ \\
\hline Skin & $0 \cdot 2302$ & $\begin{array}{l}\text { Exposure (inter) } \\
\text { Exposure (high) } \\
\text { Anxiety }\end{array}$ & $\begin{array}{l}0 \cdot 72 \\
1 \cdot 88 \\
0 \cdot 09\end{array}$ & $\begin{array}{l}0 \cdot 30 \\
0 \cdot 28 \\
0 \cdot 02\end{array}$ & $\begin{array}{l}0.0181 \\
0.0001 \\
0.0001\end{array}$ & $\begin{array}{l}0 \cdot 19 \\
0 \cdot 48 \\
0 \cdot 18\end{array}$ \\
\hline Nose & $0 \cdot 2500$ & $\begin{array}{l}\text { Exposure (inter) } \\
\text { Exposure (high) } \\
\text { Anxiety } \\
\text { Age }\end{array}$ & $\begin{array}{l}0 \cdot 87 \\
1 \cdot 60 \\
0 \cdot 10 \\
0 \cdot 01\end{array}$ & $\begin{array}{l}0 \cdot 254 \\
0 \cdot 246 \\
0 \cdot 020 \\
0.005\end{array}$ & $\begin{array}{l}0.0006 \\
0.0001 \\
0.0001 \\
0.0044\end{array}$ & $\begin{array}{l}0 \cdot 23 \\
0 \cdot 41 \\
0 \cdot 20 \\
0 \cdot 13\end{array}$ \\
\hline Breathing & 0.0257 & $\begin{array}{l}\text { Exposure (inter) } \\
\text { Exposure (high) } \\
\text { Anxiety } \\
\text { Age }\end{array}$ & $\begin{array}{l}0.91 \\
1.77 \\
0.12 \\
0.01\end{array}$ & $\begin{array}{l}0 \cdot 197 \\
0 \cdot 194 \\
0 \cdot 018 \\
0 \cdot 004\end{array}$ & $\begin{array}{l}0.0001 \\
0.0001 \\
0.0001 \\
0.0004\end{array}$ & $\begin{array}{l}0 \cdot 24 \\
0 \cdot 45 \\
0 \cdot 23 \\
0 \cdot 13\end{array}$ \\
\hline Eye & 0.9117 & $\begin{array}{l}\text { Exposure (inter) } \\
\text { Exposure (high) } \\
\text { Anxiety } \\
\text { Age } \\
\text { Predisposition }\end{array}$ & $\begin{array}{l}0.97 \\
2 \cdot 16 \\
0 \cdot 11 \\
0 \cdot 01 \\
0 \cdot 33\end{array}$ & $\begin{array}{l}0 \cdot 226 \\
0 \cdot 217 \\
0 \cdot 019 \\
0 \cdot 004 \\
0 \cdot 166\end{array}$ & $\begin{array}{l}0.0001 \\
0.0001 \\
0.0001 \\
0.0355 \\
0.0464\end{array}$ & $\begin{array}{l}0.25 \\
0.55 \\
0.22 \\
0.09 \\
0.08\end{array}$ \\
\hline Wake up & $0 \cdot 1609$ & $\begin{array}{l}\text { Exposure (inter) } \\
\text { Exposure (high) } \\
\text { Anxiety } \\
\text { Age } \\
\text { Predisposition }\end{array}$ & $\begin{array}{l}0 \cdot 64 \\
1 \cdot 65 \\
0 \cdot 13 \\
0 \cdot 01 \\
0 \cdot 38\end{array}$ & $\begin{array}{l}0 \cdot 213 \\
0 \cdot 204 \\
0 \cdot 019 \\
0 \cdot 004 \\
0 \cdot 165\end{array}$ & $\begin{array}{l}0.0025 \\
0.0001 \\
0.0001 \\
0.0012 \\
0.0199\end{array}$ & $\begin{array}{l}0.17 \\
0.42 \\
0.26 \\
0.13 \\
0.09\end{array}$ \\
\hline Miscellaneous & 0.5541 & $\begin{array}{l}\text { Exposure (inter) } \\
\text { Exposure (high) } \\
\text { Anxiety } \\
\text { Age }\end{array}$ & $\begin{array}{l}1 \cdot 56 \\
2 \cdot 28 \\
0 \cdot 21 \\
0 \cdot 03\end{array}$ & $\begin{array}{l}0 \cdot 344 \\
0 \cdot 336 \\
0 \cdot 022 \\
0 \cdot 005\end{array}$ & $\begin{array}{l}0.0001 \\
0.0001 \\
0.0001 \\
0.0001\end{array}$ & $\begin{array}{l}0.41 \\
0.58 \\
0.42 \\
0 \cdot 27\end{array}$ \\
\hline
\end{tabular}

* Results are based on stepwise logistic regression analysis.

t The dichotomous outcome is at least one severe symptom in the organ/function system continuing two years past the spill $\neq$ The design variables, Exposure (inter) and Exposure (high), were forced into models. The other independent variables offered in models were scores of Anxiety, Depression, Hostility and Paranoid, as well as Age, Gender, and Predisposition and they were selected stepwise at the 0.05 level. 
Table 5 Predictors of severe individual symptoms with individual psychological construct scores*

\begin{tabular}{|c|c|c|c|c|c|c|}
\hline $\begin{array}{l}\text { Organ/function } \\
\text { system } \dagger\end{array}$ & $\begin{array}{l}p \text { value for } \\
\text { the goodness } \\
\text { of fit }\end{array}$ & $\begin{array}{l}\text { Predictors entering } \\
\text { the model } \neq\end{array}$ & $\hat{\beta}$ & $S E(\hat{\beta})$ & $p$ & $\begin{array}{l}\text { Standardised } \\
\text { estimate }\end{array}$ \\
\hline Phlegm & 0.8531 & $\begin{array}{l}\text { Exposure (inter) } \\
\text { Exposure (high) } \\
\text { Anxiety } \\
\text { Hostility } \\
\text { Predisposition }\end{array}$ & $\begin{array}{r}0.53 \\
1.54 \\
0.13 \\
-0.15 \\
0.41\end{array}$ & $\begin{array}{l}0 \cdot 279 \\
0 \cdot 260 \\
0 \cdot 031 \\
0 \cdot 048 \\
0 \cdot 204\end{array}$ & $\begin{array}{l}0 \cdot 0562 \\
0 \cdot 0001 \\
0 \cdot 0001 \\
0 \cdot 0021 \\
0 \cdot 0468\end{array}$ & $\begin{array}{r}0 \cdot 14 \\
0 \cdot 39 \\
0 \cdot 25 \\
-0 \cdot 22 \\
0 \cdot 10\end{array}$ \\
\hline Diarrhoea & 0.4529 & $\begin{array}{l}\text { Exposure (inter) } \\
\text { Exposure (high) } \\
\text { Anxiety } \\
\text { Hostility }\end{array}$ & $\begin{array}{r}-0.08 \\
1.32 \\
0.24 \\
-0.25\end{array}$ & $\begin{array}{l}0.532 \\
0.444 \\
0.045 \\
0.078\end{array}$ & $\begin{array}{l}0 \cdot 8782 \\
0 \cdot 0030 \\
0 \cdot 0001 \\
0.0016\end{array}$ & $\begin{array}{r}-0.02 \\
0.33 \\
0.47 \\
-0.38\end{array}$ \\
\hline $\begin{array}{l}\text { Shortness of } \\
\text { breath }\end{array}$ & 0.7061 & $\begin{array}{l}\text { Exposure (inter) } \\
\text { Exposure (high) } \\
\text { Anxiety } \\
\text { Age } \\
\text { Predisposition }\end{array}$ & $\begin{array}{l}0.64 \\
2 \cdot 17 \\
0 \cdot 10 \\
0.02 \\
0.76\end{array}$ & $\begin{array}{l}0 \cdot 336 \\
0 \cdot 304 \\
0 \cdot 022 \\
0 \cdot 006 \\
0 \cdot 203\end{array}$ & $\begin{array}{l}0.0581 \\
0 \cdot 0001 \\
0 \cdot 0001 \\
0 \cdot 0001 \\
0.0002\end{array}$ & $\begin{array}{l}0 \cdot 17 \\
0 \cdot 55 \\
0 \cdot 22 \\
0 \cdot 21 \\
0 \cdot 18\end{array}$ \\
\hline Headaches & 0.4627 & $\begin{array}{l}\text { Exposure (inter) } \\
\text { Exposure (high) } \\
\text { Anxiety } \\
\text { Gender }\end{array}$ & $\begin{array}{l}0.51 \\
1.61 \\
0.13 \\
0.45\end{array}$ & $\begin{array}{l}0 \cdot 250 \\
0 \cdot 233 \\
0 \cdot 020 \\
0 \cdot 177\end{array}$ & $\begin{array}{l}0 \cdot 0426 \\
0 \cdot 0001 \\
0 \cdot 0001 \\
0 \cdot 0119\end{array}$ & $\begin{array}{l}0 \cdot 13 \\
0 \cdot 41 \\
0 \cdot 25 \\
0 \cdot 12\end{array}$ \\
\hline $\begin{array}{l}\text { Trouble } \\
\text { concentrating }\end{array}$ & 0.6494 & $\begin{array}{l}\text { Exposure (inter) } \\
\text { Exposure (high) } \\
\text { Anxiety } \\
\text { Age } \\
\text { Gender }\end{array}$ & $\begin{array}{r}1.02 \\
1.31 \\
0.30 \\
0.03 \\
-0.55\end{array}$ & $\begin{array}{l}0 \cdot 484 \\
0 \cdot 472 \\
0 \cdot 028 \\
0 \cdot 008 \\
0 \cdot 279\end{array}$ & $\begin{array}{l}0.0344 \\
0.0056 \\
0.0001 \\
0.0006 \\
0.0495\end{array}$ & $\begin{array}{r}0.27 \\
0.33 \\
0.59 \\
0.27 \\
-0.15\end{array}$ \\
\hline
\end{tabular}

* Results are based on stepwise logistic regression analysis.

tThe dichotomous outcome is severe symptom continuing two years past the spill.
The design variables, Exposure (inter) and Exposure (high), were forced into models. The other independent variables offered \$ The design variables, Exposure (inter) and Exposure (high), were forced into models. The other independent variables offered
in models were scores of Anxiety, Depression, Hostility and Paranoid, as well as Age, Gender, and Predisposition and they were in models were scores of Anxiety,
selected stepwise at the 0.05 level.

offered in the model showed that somatisation dominated the prediction as an indicator of psychological status. This was to be expected since the symptoms in the somatisation construct were most closely like the physical symptoms (see Appendix).

Analyses reported in tables 4 and 5 examine the relationship between physical symptoms and other dimensions of psychological status, that is, anxiety, depression, hostility, and paranoia. Anxiety was the best psychological indicator in predicting severe physical symptoms. The constructs depression and paranoia did not enter the final model in any of the nine organ/function systems. Analyses in table 5 show that anxiety was more prominent in explaining a severe response in diarrhoea and trouble concentrating than was the high $\mathrm{HF}$ exposure.

\section{Discussion}

Disasters can have both physical and psychological effects in those exposed. ${ }^{16-1924}$ The results presented here, in conjunction with those presented in Dayal et al, ${ }^{15}$ support the psycho-biological paradigm of health sequelae of a disaster involving a community exposure to HF. Based on self reported information, we found that high exposure to a chemical release disaster had a long term (more than two years) impact on physical health which could not be explained by psychological status. The extent to which the effect of the psychological status varied across symptoms, however, was noteworthy. For those organ/function systems more likely to be directly related to the HF exposure (skin, breathing (by inhalation), throat), the impact of psychological variables was much less pronounced than that of the high $\mathrm{HF}$ exposure. For these systems, however, the effect of the intermediate level of exposure was comparable with that of psychological distress. The psychological status was weighted more heavily among those physical symptom groups (stomach, wake up, miscellaneous) more often associated with psychological stress. This pattern of response tends to reinforce the idea that some of the symptom reports were not psychological in aetiology, while others had a substantial psychological status component.

The list of symptoms used to assess psychological status came from the validated BSI. We retained in its entirety a subset of symptoms associated with five constructs. This is a reasonable way of obtaining an instrument of manageable length when interest is limited to a subset of constructs. The consistency and interpretability of findings affirm the appropriateness of the symptom list. The published reports on the psychological effects of environmental stress indicate little uniformity over the instruments used. ${ }^{25}$ For instance, Horwitz and Stefanko ${ }^{26}$ adapted the SCL-90 for their study of the psychological effects of living near a toxic waste landfill by adding questions to the somatisation subscale, using a demoralisation subscale from another instrument, and modifying it in other ways to reduce the number of items so as to keep the total survey to a reasonable length. Given the variety of contexts in which psychological status needs to be measured and the pressing extrascientific considerations, investigators have to devise instruments to assess psychological variables. The lack of uniformity makes it difficult to compare findings and interpretations, except at the conceptual level.

There are inherent issues of direction of causality when inferences are drawn from a cross sectional survey, especially if a questionnaire is used to assess both exposure and health at the same time. ${ }^{27}$ Flanders et $a l^{27}$ suggested that a 'checkmark' pattern - that is, an inconsistent pattern in which disease prevalence is highest in the designated exposed group, but is smaller in the internal than in the external control group, must be investigated 
carefully for the true direction of causality. Environmental studies do not typically include additional control groups; the Texas City study did not include one either. The control group came from the same neighborhoods as those with high $\mathrm{HF}$ exposure, thereby controlling for baseline physical and psychological symptoms. The observed associations in the present study were always consistent with a strong dose response, the primary criterion for causality in Flanders et al. ${ }^{27}$ Moreover, the exposure and outcome assessments were not done at the same time: the two were separated by approximately one year and used different methods for collecting the information.

The findings in this report are based on a symptom prevalence study which relies on self report. Symptom reports are known to be limited by methodological considerations of reliability, differential recall bias, ${ }^{28} 29$ behavioral sensitisation, ${ }^{30-32}$ and validity. ${ }^{33}$ Reproducibility is a good indicator of reliability. In interviewreinterview of a $10 \%$ subsample, the agreement in data on severe symptoms was over $85 \%$. Differential recall bias and behavioural sensitisation are closely related concepts dealing with the phenomenon of cases (exposed) recalling and reporting events differently from controls (unexposed). This occurs in many studies because the two groups come from different populations and, therefore, do not share the life disturbing experience. However, in this study, it was possible to obtain an internal unexposed group from the same community because of two factors: (1) the HF plume dispersal did not cover the entire town, and (2) many people at the same time of the release were not at home in the area affected by the HF cloud, and, conversely, some people were going home from work through the cloud and were exposed even though their homes were not in the affected area. Since the cases and controls in the Texas City study came from the same community and, therefore, had comparable background effects from living in the chronically exposed community, and had partially comparable experiences of the $\mathrm{HF}$ release as a disrupter of their lives, the effect of differential recall bias and behavioural sensitisation should have been minimised.

Compensation neurosis is the neurotic phenomenon that develops after an accident in people who are insured or who believe that by being ill they may increase their chances of receiving compensation. It is possible that some of the results of our analysis are attributable to compensation neurosis. The following points must be considered, however, in judging the likelihood that this phenomenon may have rendered the findings invalid. In our survey, many people refused to participate because their legal advisors had advised them to do so. A study in which compensation neurosis is an important factor would be expected to yield a high percentage of people reporting high exposure and severe symptoms. Also, data from such a study would be expected to show that a high percentage of highly exposed people continued severe symptoms into the future. In our study, only $6.7 \%$ individuals were de- termined to have been heavily exposed. Moreover, only 20 to $30 \%$ of the individuals in the highly exposed group reported continuing severe problems related to breathing two years past the spill. Thus, although it is difficult to dismiss compensation neurosis as a possible confounder, it seems the findings of this study can not be explained by such phenomenon.

Whether the symptoms we selected as indicative of HF exposure were truly pathogonomonic of this can be questioned. However, while other severe illnesses could lead to these symptoms, it should be noted that because of the large sample, we were able to balance the three exposure groups in terms of conditions that might predispose to such symptoms - for example, smoking and chronic lung disease. Similarly, it might be suggested that our findings are due to the sort of person who likes to say "yes" answering "yes" to exposure assessment and to outcome assessment symptoms. However, as noted earlier, the exposure and outcome assessments were chronologically separated by approximately one year and used different methods for collecting the information. It should also be noted that the psychological symptoms were not identified as such, and, therefore, there is no reason to believe that the "yes saying" people would answer these questions differently from the others. The pattern of results in which covariance analyses with psychological symptoms minimise the effect of exposure for symptoms often considered psychological, but not for symptoms considered more likely to be directly related to an $\mathrm{HF}$ exposure, mitigates against a "yes saying" bias.

In theory, the validity of self reported symptoms can be ascertained by verifying medical records or performing clinical tests on the respondents. However, such an approach in a large community setting is not feasible. We adopted a practical approach to validating the observed association between exposure and reported symptoms by seeking corroboration with the open-ended data on names of medications reported to have been taken by the respondents. ${ }^{34}$ The medication use for an HF related problem was found to be associated with the exposure level. Moreover, there was a uniformly strong association between systemspecific medication use and the severity of symptoms at each level of exposure. These findings provide an indirect measure of validity for symptom reports as well as for exposure.

Inferences from this and an earlier paper ${ }^{15}$ hinge on the assignment of individuals to exposure categories. Exposure assessment was partly based on responses to three acute symptoms in the study undertaken one year afterwards, while the outcome assessment was based on short and long term symptom prevalence in the symptom assessment study two years afterwards. Using symptom reports partly to define exposure and assess outcome (even though at different times), complicates the attribution of excess severe symptoms to the true exposure. However, the following points are noteworthy: (i) the three symptoms that were considered in the exposure assessment are 
pathognomonic of an acute $\mathrm{HF}$ exposure; (ii) the outcome symptoms included far more than three symptoms; (iii) as noted earlier, the three pathognomic symptoms and the 40 outcome symptoms were assessed at different times (a year apart) and in different ways: and, most importantly, (iv) the outcome symptoms reported by individuals placed in the highly exposed category resembled closely those reported by the special group of individuals included in the study who were known to have received relatively higher doses of exposure (that is, the police officers managing the evacuation and the cases admitted to hospital after the accident ${ }^{15}$ ).

Our pattern of psychosocial impact results conforms to that found by others. Several authors found that the primary response to an emergency was the somatisation scale, followed by the anxiety scale, ${ }^{1835}$ lending validity to the pattern of our findings. A number of studies have shown that higher levels of anxiety are associated with more symptom reporting. ${ }^{3637}$ Pennebake ${ }^{20}$ has interpreted this to mean that highly anxious people are more attentive to symptoms. Schwartz et $a^{37}$ showed that both cognitive and somatic anxiety are correlated with symptom reporting, somatic more so than cognitive. Our findings are consistent with these observations.

The results in table 5 show a positive correlation between anxiety and diarrhoea (as might be expected), but a negative association between hostility and diarrhoea. Several interpretations may be proposed for the negative relation. It is possible that hostile people do not ordinarily report this symptom; that hostile people often have diarrhoea and do not attribute it to an external event; or, that hostile people get more immediate attention for such a symptom, thereby minimising its duration and the memory of it. We know of no empirical evidence which would help select from among these alternatives explanations.

Communities in which studies of health sequelae to a man made disaster are to be conducted may feel that the psychological symptom data may be used against them in a "blame the victim" manner. That is, they may feel that their reported physical problems may be attributed to their psychological stress, thereby exonerating the physical insult from the disaster as the cause. It can be justifiably argued, however, that if no attempt is made to assess the psychological component, the true physical and psychological impact of the exposure may never be known. Collecting data on psychological variables, especially on somatic and cognitive anxiety, seems to be important in future studies of the effects of accidental exposures on the health of a community.

\section{Appendix}

LIST OF 33 PSYCHOLOGICAL SYMPTOMS IN THE QUESTIONNAIRE

Anxiety

Nervousness or shakiness inside

Suddenly scared for no reason
Feeling fearful

(Q14)

Feeling tense

(Q23)

Spells of terror

(Q27)

Feeling so restless you couldn't sit still (Q30)

Depression

Thoughts of ending your life

(Q6)

Feeling lonely

Feeling blue

Feeling no interest in things

(Q11)

(Q12)

(Q13)

Feeling hopeless about the future

(Q21)

Feelings of worthlessness

(Q31)

Hostility

Feeling easily annoyed or irritated

Temper outbursts that you could not control

Having urges to beat, injure, or harm someone

Having urges to break or smash things

Getting into frequent arguments

(Q25)

(Q26)

Paranoia

Feeling others are to blame for most of your troubles

Feeling that most people can not be trusted

Feeling that you are watched or talked about by others

Others not giving you proper credit for your achievements

Feeling that people will take advantage of you if you let them

Somatisation

Faintness or dizziness

Pains in heart or chest

Nausea or upset stomach

Trouble getting your breath

Hot or cold spells

(Q19)

Numbness or tingling in parts of your body

Feeling weak in parts of your body

Miscellaneous

Poor appetite

Trouble falling asleep

Thoughts of death or dying

Feelings of guilt

The study was partially supported by funds from the Galveston County Health District and the Agency for Toxic Substance Disease Registry, Public Health Services.

1 Occupational Safety and Health Administration. Occupational health guideline for hydrogen fluoride. Washington: US Department of Health and Human Services, September 1978 .

2 Caravati E. Acute hydrofluoric acid exposure. Am 7 Emerg Med 1988;6:143-50.

3 Hileman B. Fluoridation of water. Special report, Chemical and Engineering News 1988;66:26-42.

4 Krishnamachari $\mathrm{K}$. Skeletal fluorosis in humans. A review of recent progress in understanding the disease. Prog Food Nutr Sci 1986;10:279-314.

5 Tamboli BL, Mathur GM. Clinical criteria for screening of skeletal fluoriosis in an endemic area. Indian $f$ Public Health 1982;26:244-9.

6 Mayer L, Geulich J. Hydrogen fluoride (HF) inhalation and burns. Arch Environ Health 1963;7:445-7.

7 Kleinfield $M$. Acute pulmonary edema of chemical origin. Arch Environ Health 1965;10:942-46.

8 Dieffenbacher P, Thompson J. Burns from exposure to anhydrous hydrofluoric acid. 7 Occup Med 1962;4:325-6.

9 Braun J, Stob H, Zober A. Intoxication following the inhalation of hydrogen fluoride. Arch Toxicol 1984;56:50-4.

10 Shewmake S, Anderson B. Hydrofluoric acid burns: a report of a case and review of the literature. Arch Dermatol 1979; 115:593-6.

11 Dale R. Treatment of hydrofluoric acid burns. BMf 1951; i: $728-32$.

12 Tepperman P. Fatality due to acute systemic fluoride poisoning following a hydrofluoric acid skin burn. $f$ Occup Med 1980;22:691-2. 
13 Mayer T, Gross P. Fatal systemic fluorosis due to hydrofluoric acid burns. Ann Emerg Med 1985;14:149-53.

14 MeIvor M. Delayed fatal hyperkalemia in a patient with acute fluoride intoxication. Ann Emerg Med 1987;16:11657

15 Dayal H, Brodwick M, Morris R, Baranowski T, Trieff $\mathrm{N}$, Harrison J, Lisse J, Ansari G. A community-based epidemiologic study of health sequelae of exposure to hydrofluoric acid. Ann Epidemiol 1992;2:213-30.

16 Kinston W, Rosser R. Disaster: Effects on mental and physical state. $\mathcal{F}$ Psychosom Res 1974;18:437-56.

17 Logue J, Melick M, Hansen H. Research issues and directions in the epidemiology of health effects of disasters. Epidemiol Rev 1981;3:140-62.

18 Baum A, Gatchel RJ, Schaeffer MA. Emotional, behavioral, and physiological effects of chronic stress at Three Mile Island. $\mathcal{F}$ Consult Clin Psychol 1983;51:565-72.

19 Davidson LM, Baum A. Chronic stress and posttraumatic stress disorders. 7 Consult Clin Psychol 1986;54:303-8.

20 Pennebaker J. The psychology of physical symptoms. New York: Springer Verlag, 1982.

21 Derogatis LR. SCL-90-R. Administration, scoring and procedures manual-I. Baltimore MD: Johns Hopkins Unicedures manual-I. Baltimore MD
versity School of Medicine, 1977 .

22 Derogatis L, Melisaratos N. The brief symptom Inventory: an introductory report. Psychol Med 1983;13:595-605

23 Hosmer DW, Lemeshow S. Applied logistic regression. New York: John Wiley, 1989

24 Hartsough D, Savitsky J. Three Mile Island: Psychological and environmental policy at a crossroad. Am Psychol 1984; 39:1113-22.

25 Rubonis AV, Bickman L. Psychological impairment in the wake of disaster: The disaster-psychopathology relationship. Psychol Bull 1991;109:384-99.

26 Horwitz J, Stefanko M. Toxic waste: Behavorial effects of an environmental stressor. Behav Med 1989;15(1):23-8.

27 Flanders WD, Lin L, Pirkle JL, Caudill SP. Assessing the direction of causality in cross-sectional studies. $A m \mathcal{F}$ Epidemiol 1992;135(8):926-35.
28 Raphael, K. Recall bias: A proposal for assessment and control. Int f Epidemiol 1987;16:167-70.

29 Roht LH, Vernon SW, Weir FW et al. Community exposure to hazardous waste disposal sites: Assessing reporting bias. Am $\mathcal{F}$ Epidemiol 1985;122:418-33.

30 Neutra R, Lipscomb JA, Satin KP, Shusterman D. Factors which might contribute to higher symptom rate sometimes observed around hazardous waste sites. Proceedings of the 2nd Annual Meeting of the International Society of Envinnmental Epidemiology (ISEE), University of California, Berkeley, August 12-15, 1990.

31 Mendell MG, Lipscomb J. Interpretation of symptom data in populations with environmental worry. Proceedings of the 2nd Annual Meeting of the International Society of Environmental Epidemiology (ISEE). University of California, Berkeley, August 12-15, 1990.

32 Shusterman D, Lipscomb J, Neutra R, Satin K. Symptom prevalence and odor-worry interaction near hazardous prevalence and odor-worry interaction near hazardous
waste sites. Proceedings of the 2nd Annual Meeting of the International Society of Environmental Epidemiology (ISEE).

33 Ozonoff D, Colten ME, Cupples A, et al. Health problems reported by residents of a neighbourhood contaminated reported by residents of a neighbourhood contaminated by a hazar.

34 Dayal H, Li Y, Dayal V, Mittal C, Snodgrass W. Use of medication data to validate the finding of an association in community-based symptom prevalence studies. Arch Env Health 1994; 49(2): 93-7.

35 Gatchel RJ, Schaeffer MA, Baum A. A psychophysiological field study of stress at Three Mile Island. Psychophysiology 1985;22:175-181.

36 Lipman R, Rickels K, Covi L, Derogatis L, Uhlenhuth E. Factors of symptom distress. Arch Gen Psychiatr 1969;21: 328-38.

37 Schwartz GE, Davidson RJ, Goleman DJ. Patterning of cognitive and somatic processes in the self-generation of anxiety: Effects of meditation versus exercise. Psychosom Med 1978;40:321-8. 